\title{
Collisional decoherence of polar molecules
}

\author{
Kai Walter, Benjamin A. Stickler, and Klaus Hornberger \\ Faculty of Physics, University of Duisburg-Essen, Lotharstraße 1, 47048 Duisburg, Germany
}

\begin{abstract}
The quantum state of motion of a large and rotating polar molecule can lose coherence through the collisions with gas atoms. We show how the associated quantum master equation for the centerof-mass can be expressed in terms of the orientationally averaged differential and total scattering cross sections, for which we provide approximate analytic expressions. The master equation is then utilized to quantify collisional decoherence in a interference experiment with polar molecules.
\end{abstract}

PACS numbers: 03.65.Yz,34.50.-s,03.75.-b

\section{INTRODUCTION}

Coherence experiments with molecules and molecular clusters test the quantum superposition principle and allow measurement of molecular vacuum properties with unprecedented accuracy [14]. Performing such experiments with organic molecules or biomolecules [5] 8], which are predominantly polar, raises the question of how their quantum coherence is affected by collisions with a background gas. It is the aim of the current article to clarify the influence of molecular dipole moments on collisional decoherence due to scattering with gas atoms.

Collisional decoherence is ubiquitous, its relevance reaching well beyond matter-wave experiments 9 14]. While it is still unclear whether the quantum superposition principle is valid at all scales [15-17, the effect of decoherence due to scattering with environmental particles provides an experimentally tested quantum description of the appearance of classical dynamics [4, 18, 19].

In the case of a spherical, polarizable particle, the quantum linear Boltzmann equation gives a Markovian, nonperturbative microscopic description of the particle's motion through a thermal environment [20, 21]. If the molecule is much heavier than the gas particle, this master equation can be expressed in terms of a scattering rate and a decoherence function. These quantities are determined by the total and the differential scattering cross section of a single collision, as calculated using standard scattering theory [22].

In order to formulate the theory of collisional decoherence for nonspherical particles, one must take the molecule's center of mass as well as its orientational degrees of freedom into account. In general, this implies solving the full inelastic scattering problem to obtain the scattering amplitudes required for the master equation [12, 23, 24]. Yet, if the molecule is sufficiently massive and approximately static during a single collision, the scattering event is effectively elastic [25]. We will see that the decoherence function and the scattering rate then depend on the orientationally averaged cross sections. Here, we can draw on the orientation-dependent cross sections, that were calculated and measured in various molecular collision experiments [25 28]. We extend these works and derive the resulting decoherence function and scattering rate. It is shown that both quantities can be measured by placing a collision chamber in a matterwave interferometer. Comparison of the scattering rate of two different polar molecules provides a nonspectroscopic means to measure their relative electric dipole moment in the gas phase.

We note that our analytic expressions for the orientation-dependent total and differential cross sections may also become relevant for future experiments where quantum coherence plays no role. This includes buffer gas cooling of large polar molecules [29, 30] and dedicated collision experiments with molecular beams [31. Moreover, they can be used to assess the influence of background gases in molecular beam deflection measurements 32 34.

The paper is structured as follows: In Sec. II we present the master equation for the center-of-mass degrees of freedom of a slowly rotating molecule in a homogeneous gas environment. Section III considers the scattering between a non-spherical particle and a single gas particle in order to obtain approximate expressions for the total as well as the differential scattering cross section, and in Sec. IV we derive the decoherence function and the scattering rate. In Sec. V collisional decoherence in a typical far-field matter-wave setup is studied as an application. We conclude in Sec. VI]

\section{MASTER EQUATION FOR THE CENTER-OF-MASS MOTION}

We consider a rigid top molecule of mass $M$ which enters with velocity $\mathbf{v}_{\mathrm{M}}$ a chamber filled with a monoatomic gas at temperature $T$. The molecular degrees of freedom (DOFs) are its center-of-mass (c.m.) position $\mathbf{R}$ and its orientation $\Omega$, specified for instance by the Euler angles in the $z-y^{\prime}-z^{\prime \prime}$-convention [35, 36]. Since the gas is at thermal equilibrium, the state of the gas $\rho_{\mathrm{g}}$ is diagonal in the momentum basis and its diagonal elements are given by the Boltzmann distribution $\mu(|\mathbf{p}|)$.

For isotropic interaction potentials a Markovian master equation can be derived by means of the so-called monitoring approach [37, 38]. The resulting equation is then characterized by the rate operator $\Gamma$ as well as by the scattering operator $\mathrm{S}$, which describes the modification of the composite molecule-gas state $\rho_{\text {tot }}$ by a single 
scattering event, $\rho_{\text {tot }} \rightarrow \rho_{\text {tot }}^{\prime}=\mathrm{S} \rho_{\text {tot }} \mathrm{S}^{\dagger}$.

In the case of non-spherical molecules, the interaction potential is anisotropic and the orientation state must be taken into account. This leads in general to a multichannel scattering problem [23] where the Schrödinger equation must be solved for arbitrary initial and final rotation states of the molecule [39. However, when considering the room-temperature collision between a multiatomic molecule and a fast single atom, the rotational period is typically much smaller than the collision time and the molecule can be assumed to be rotationally static during the scattering process (sudden approximation) [25]. The scattering operator as well as the rate operator are then diagonal in the orientational DOFs since the Schrödinger equation depends only parametrically on the orientation [40].

Here, we are only interested in the c.m. dynamics of the molecule and, thus, trace out the environmental as well as the orientational DOFs. It follows from the monitoring approach 23, 37] that the motional state of a slowly rotating molecule in a gaseous environment is described by a master equation

$$
\partial_{t} \rho=\frac{1}{i \hbar}[\mathrm{H}, \rho]+\mathcal{R} \rho+\mathcal{L} \rho,
$$

where $\mathrm{H}=\mathrm{P}^{2} / 2 M$ is the free Hamiltonian. The two superoperators in (1) can be given as

$$
\begin{aligned}
\mathcal{R} \rho= & i\left\langle\operatorname{Tr}_{\mathrm{g}}\left(\left[\Gamma^{1 / 2} \operatorname{Re}(\mathrm{T}) \Gamma^{1 / 2}, \rho \otimes \rho_{\mathrm{g}}\right]\right)\right\rangle, \\
\mathcal{L} \rho= & \left\langle\operatorname { T r } _ { \mathrm { g } } \left(\mathrm{T} \Gamma^{1 / 2} \rho \otimes \rho_{\mathrm{g}} \Gamma^{1 / 2} \mathrm{~T}^{\dagger}\right.\right. \\
& \left.\left.-\frac{1}{2}\left\{\rho \otimes \rho_{\mathrm{g}}, \Gamma^{1 / 2} \mathrm{~T}^{\dagger} \mathrm{T} \Gamma^{1 / 2}\right\}\right)\right\rangle,
\end{aligned}
$$

where $\mathrm{T}=\mathrm{T}(\Omega)$ is the non-trivial part of the scattering operator, $\mathrm{S}(\Omega)=1+i \mathrm{~T}(\Omega)$, and the expectation value of the rate operator $\Gamma=\Gamma(\Omega)$ gives the scattering rate. Here, the angular brackets $\langle\cdot\rangle$ denote the orientational average with respect to the rotation state of the molecule. We note that the orientational coherences $\left\langle\Omega\left|\rho_{\mathrm{M}}\right| \Omega^{\prime}\right\rangle$ do not contribute to the master equation (1) because the rate operator and the scattering operator are diagonal in the orientational DOFs.

In most experimentally relevant cases the initial rotation state is thermal, implying that the orientational DOFs are homogeneously distributed. Since the gas distribution $\mu(|\mathbf{p}|)$ is approximately isotropic, it is natural to assume that the orientational distribution remains homogeneous for all times, i.e. the orientational diagonal elements of the total molecular state $\rho_{\mathrm{M}}$ are $\left\langle\Omega\left|\rho_{\mathrm{M}}\right| \Omega\right\rangle=\rho /\left(8 \pi^{2}\right)$. Then the orientational average of the function $K(\Omega)$ is

$$
\langle K(\Omega)\rangle=\int_{S} \frac{d \Omega}{8 \pi^{2}} K(\Omega),
$$

with $S=S_{2} \times S_{1}$ being the configuration space of the orientational DOFs.
The fact that the molecular mass $M$ fairly exceeds the mass $m$ of a gas atom allows us to further simplify Eq. (1). In the limit that $m / M$ vanishes, Eq. (2) gives a constant energy shift [21], which can be ignored,f and the second term (3) describes decoherence in the position representation [21, 38,

$$
\begin{aligned}
\lim _{m / M \rightarrow 0}\left\langle\mathbf{R}_{1}|\mathcal{L} \rho| \mathbf{R}_{2}\right\rangle= & -\gamma\left[1-\eta\left(\mathbf{R}_{1}-\mathbf{R}_{2}\right)\right] \\
& \times\left\langle\mathbf{R}_{1}|\rho| \mathbf{R}_{2}\right\rangle .
\end{aligned}
$$

This expression can be derived following the same steps [21] as for a point particle. The scattering rate $\gamma$ in (5) can be given as

$$
\gamma=\frac{n_{\mathrm{g}}}{m} \int d^{3} \mathbf{p} p \mu\left(\left|\mathbf{p}+m \mathbf{v}_{\mathrm{M}}\right|\right)\left\langle\sigma_{\mathrm{tot}}(p)\right\rangle,
$$

where $p=|\mathbf{p}|$ is the length of the momentum vector and $n_{\mathrm{g}}$ is the density of the gas. Equation (5) also involves the decoherence function

$$
\begin{aligned}
\eta(\mathbf{R})= & \frac{1}{N} \int_{0}^{\infty} d p p^{3} \int_{S_{2}} d^{2} \mathbf{n} \int_{S_{2}} d^{2} \mathbf{n}^{\prime} \mu\left(\left|\mathbf{p}+m \mathbf{v}_{\mathrm{M}}\right|\right) \\
& \times\left\langle\left|f\left(p, \mathbf{n} \cdot \mathbf{n}^{\prime}\right)\right|^{2}\right\rangle \exp \left(\frac{i p}{\hbar}\left(\mathbf{n}-\mathbf{n}^{\prime}\right) \cdot \mathbf{R}\right),
\end{aligned}
$$

where $\mathbf{n}$ and $\mathbf{n}^{\prime}$ are the incoming and outgoing directions of the scattered gas atom, respectively, and $S_{2}$ denotes the surface of the unit sphere. Here, the normalization constant $N$ ensures that $\eta(0)=1$, so that the diagonal elements of $\rho$ are preserved by Eq. (5). The fact that the differential scattering cross section $\left\langle\left|f\left(p, \mathbf{n} \cdot \mathbf{n}^{\prime}\right)\right|^{2}\right\rangle$ depends on the angle between incoming and outgoing momentum rather than the individual directions is due to the trace over the orientational DOFs performed to derive Eq. (1). The Fourier transform of the decoherence function $\eta(\mathbf{R})$ gives the probability distribution of transferred momentum of a single collision [41].

Note that the thermal gas distribution in Eqs. (6) and 77 is shifted by $m \mathbf{v}_{\mathrm{M}}$ due to the molecule's velocity. Since the most probable momentum $p_{\mathrm{g}}=\sqrt{2 m k_{\mathrm{B}} T}$ in a thermal gas distribution $\mu(p)$ is usually much higher than the momentum $m v_{\mathrm{M}}$, Eqs. (6) and (7) can be expanded in orders of $m v_{\mathrm{M}} / p_{\mathrm{g}}$. This yields

$$
\gamma=\frac{4 \pi n_{\mathrm{g}}}{m} \int_{0}^{\infty} d p p^{3} \mu(p)\left\langle\sigma_{\mathrm{tot}}(p)\right\rangle\left[1+\mathcal{O}\left(\frac{m^{2} v_{\mathrm{M}}^{2}}{p_{\mathrm{g}}^{2}}\right)\right],
$$

and

$$
\begin{aligned}
\eta(\mathbf{R})= & \frac{8 \pi^{2}}{N} \int_{0}^{\infty} d p p^{3} \mu(p) \int_{0}^{\pi} d \theta \sin \theta\left\langle|f(p, \cos \theta)|^{2}\right\rangle \\
& \times \operatorname{sinc}\left[\sin \left(\frac{\theta}{2}\right) \frac{2 p|\mathbf{R}|}{\hbar}\right]\left[1+\mathcal{O}\left(\frac{m^{2} v_{\mathrm{M}}^{2}}{p_{\mathrm{g}}^{2}}\right)\right],(9)
\end{aligned}
$$

with $\cos \theta=\mathbf{n} \cdot \mathbf{n}^{\prime}$. It is demonstrated in Appendix A that the linear order vanishes.

In what follows, we will specify the scattering rate $\gamma$ and the decoherence function $\eta(\mathbf{R})$ for the case of a polar or anisotropically polarizable molecule scattering with polarizable spherical particles. 


\section{CROSS SECTIONS FOR POLAR MOLECULE-ATOM SCATTERING}

It is the aim of this section to evaluate the total and the differential scattering cross sections for the collision between a gas atom and a polar molecule. Although we are mainly interested in the dipole-induced dipole interaction, we consider the more general homogeneous potential

$$
V(r, \cos \Theta)=-\frac{C}{r^{s}}\left(1+a \cos ^{2} \Theta\right),
$$

which also includes the anisotropic van der Waals interaction [42]. Here, $\Theta$ is the angle between the molecule's orientation $\mathbf{m}(\Omega)$ and the relative c.m. coordinate $\mathbf{r}$, i.e. $\cos \Theta=\mathbf{m} \cdot \mathbf{r} / r$, and $r=|\mathbf{r}|$ is the distance between the gas atom and the c.m. of the molecule. The parameter $a \geq 0$ quantifies the anisotropy of the interaction potential and $C$ is the interaction strength. For example, the dipole-induced dipole interaction is described by $s=6$ and $C=\alpha_{0} d_{0}^{2} / 32 \pi^{2} \varepsilon_{0}^{2}$ with $a=3$, where $\alpha_{0}$ is the atomic polarizability and $d_{0}$ is the molecular dipole moment. In this case $\mathbf{m}$ is the orientation of the dipole moment.

The total and the differential scattering cross sections for a fixed molecular orientation $\mathbf{m}$ can be calculated in the eikonal approximation, also referred to as Schiff's approximation [43]. This approximation provides reliable results for small angle scattering [25], i.e. for soft collisions, which we will show to be most important for the decoherence function. Choosing the initial relative momentum along the $z$-axis, $\mathbf{p}=p \mathbf{e}_{z}$, the orientation dependent scattering amplitude can be expressed as [43]

$$
f\left(p, \mathbf{n}^{\prime} ; \mathbf{m}\right)=-\mathrm{i} \frac{p}{2 \pi \hbar} \int_{\mathbb{R}^{2}} \mathrm{~d}^{2} \mathbf{b} e^{-\mathrm{i} p \mathbf{n}_{\perp}^{\prime} \cdot \mathbf{b} / \hbar}\left(\exp \left[-\frac{\mathrm{i} m}{\hbar p} \int_{-\infty}^{\infty} \mathrm{d} z V\left(\sqrt{b^{2}+z^{2}}, \frac{\left(\mathbf{b}+z \mathbf{e}_{z}\right) \cdot \mathbf{m}}{\sqrt{b^{2}+z^{2}}}\right)\right]-1\right),
$$

where $\mathbf{b}$ is the impact vector in the $x y$-plane and $\mathbf{n}_{\perp}^{\prime}$ are the $x y$-components of the direction of outgoing momentum $p \mathbf{n}^{\prime}$. Here, the scattering phase is calculated by integrating the interaction potential for a fixed molecular orientation $\mathbf{m}$ along the straight trajectory of the gas particle.

The total scattering cross section can be obtained by evaluating (11) in the forward direction, $\mathbf{n}^{\prime}=\mathbf{e}_{z}$, according to the optical theorem. The orientationally averaged total cross section is then given by [25]

$$
\left\langle\sigma_{\mathrm{tot}}(p)\right\rangle=\sigma_{0}(p) \int_{S_{2}} \frac{d^{2} \mathbf{m}}{4 \pi}\left[1+\frac{a}{s}\left(\mathbf{m} \cdot \mathbf{e}_{z}\right)^{2}+\frac{a(s-1)}{s}\left(\mathbf{m} \cdot \mathbf{e}_{x}\right)^{2}\right]^{2 /(s-1)},
$$

where

$$
\sigma_{0}(p)=2 \pi \sin \left(\frac{\pi}{2} \frac{s-3}{s-1}\right) \Gamma\left(\frac{s-3}{s-1}\right)\left(\frac{\sqrt{\pi} m C}{\hbar p} \frac{\Gamma[(s-1) / 2]}{\Gamma(s / 2)}\right)^{2 /(s-1)}
$$

is the cross section resulting from the isotropic part of the interaction potential, i.e. for $a=0$ in Eq. 10] [44. The orientational average in Eq. (12) can be well approximated (see Appendix B), and one obtains the compact expression

$$
\left\langle\sigma_{\text {tot }}(p)\right\rangle=\sigma_{0}(p)\left(1+\frac{a}{3}\right)^{2 /(s-1)}
$$

Thus the anisotropy of the potential 100 enhances the total cross section by a constant factor.

We now turn our attention to the differential scattering cross section. In general, the scattering amplitude (11) cannot be evaluated in closed form. However, for small angle scattering events [28], for which $\mathbf{n}^{\prime} \simeq \mathbf{n}$, the scattering amplitude can be evaluated by expanding the plane wave in Eq. (11) up to second order in $\mathbf{n}_{\perp} \cdot \mathbf{b}$. A straightforward derivation, presented in Appendix $B$, gives the orientation averaged differential scattering cross section

$$
\left\langle|f(p, \cos \theta)|^{2}\right\rangle=A(p)\left[1-\left(\frac{\theta}{\theta_{*}(p)}\right)^{2}+\mathcal{O}\left(\theta^{4}\right)\right]
$$

where, making the same approximations as above, we abbreviated

$$
\begin{aligned}
A(p) & =\left(\frac{p\left\langle\sigma_{\mathrm{tot}}(p)\right\rangle}{4 \pi \hbar \cos [\pi /(s-1)]}\right)^{2}, \\
\theta_{*}(p) & =\frac{\hbar}{p} \sqrt{\frac{8 \pi}{\left\langle\sigma_{\mathrm{tot}}(p)\right\rangle} \Gamma\left(\frac{s-3}{s-1}\right) \Gamma\left(\frac{s-5}{s-1}\right)^{-1 / 2} .}
\end{aligned}
$$

Thus, the differential cross section as a function of $\theta$ decays quadratically in the forward direction. However, its functional dependence for all $\theta \in[0, \pi]$ is required for the calculation of the decoherence function (7). One possibility to overcome this is to approximate the cross section by a Gaussian curve with amplitude $A \sqrt{16}$ and 
width $\theta_{*} 17$ 28

$$
\left\langle|f(p, \cos \theta)|^{2}\right\rangle \approx A(p) \exp \left[-\left(\frac{\theta}{\theta_{*}(p)}\right)^{2}\right] .
$$

This is certainly a crude approximation, and even though it was demonstrated [28, that Eq. (18) gives reliable results for soft scattering, $\theta \ll 1$, it does not contain the correct asymptotic behavior $\left\langle|f|^{2}\right\rangle \propto \theta^{-2(s+1) / s}$ for hard scattering $\theta \lesssim 1$ [44, 45]. However, the important properties of the decoherence function, i.e.,its width and its asymptotic behavior as $|\mathbf{R}| \rightarrow \infty$, are mainly determined by soft collisions, since hard collisions cause almost complete decoherence and are therefore mainly captured by the scattering rate. Importantly, this scattering rate is determined without the small-angle approximation; see Eq. (12). We remark that the differential cross section can also show oscillating behavior (glory and rainbow effect) [46] and various resonances [47, but neglecting these is justified, since they average out when evaluating the decoherence function 7 .

Having discussed the validity of the approximations used, we are now in the position to compute the decoherence function (7) and the scattering rate (6) in the next section.

\section{DECOHERENCE FUNCTION AND SCATTERING RATE}

In order to calculate the scattering rate, we insert the total scattering cross section (14) into Eq. (6) and obtain for the leading order contribution in $m v_{\mathrm{M}} / p_{\mathrm{g}}$

$$
\gamma=\frac{2 n_{\mathrm{g}} p_{\mathrm{g}}}{m \sqrt{\pi}} \Gamma\left(\frac{2 s-3}{s-1}\right)\left\langle\sigma_{\mathrm{tot}}\left(p_{\mathrm{g}}\right)\right\rangle .
$$

As expected, the scattering rate is proportional to $n_{\mathrm{g}} p_{\mathrm{g}} \sigma_{\mathrm{tot}} / m$, which is the effective current of gas atoms passing through the total scattering cross section. For the case of the dipole-induced dipole interaction, $s=6$, the rate is given by

$$
\gamma_{\mathrm{d}-\mathrm{id}}=\frac{2^{7 / 5}}{\sqrt{\pi}} \Gamma\left(\frac{9}{5}\right) \frac{n_{\mathrm{g}} p_{\mathrm{g}} \sigma_{0}\left(p_{\mathrm{g}}\right)}{m} .
$$

In particular, it is proportional to $n_{\mathrm{g}} \alpha_{0}^{2 / 5} d_{0}^{4 / 5}(T / m)^{3 / 10}$ and thus depends only weakly on the gas temperature $T$. In comparison to the scattering rate of a spherical molecule 41, one observes an additional prefactor of $2^{2 / 5} \simeq 1.3$ due to the anisotropic contribution to the interaction potential 10 .

The evaluation of the decoherence function $\eta(\mathbf{R})$ is slightly more complicated. In accordance with the small angle approximation utilized in the calculation of the differential scattering cross section (18), we replace in Eq. (7) $\sin \theta$ by $\theta$ and extend the integration boundary to infinity. Thus, the leading order contribution in $m v_{\mathrm{M}} / p_{\mathrm{g}}$ is

$$
\eta(\mathbf{R})=\int_{0}^{\infty} d \xi \nu(\xi) D\left[\frac{p_{\mathrm{g}}|\mathbf{R}| \theta_{*}\left(p_{\mathrm{g}}\right)}{2 \hbar} \xi\right],
$$

where $\xi=\left(p / p_{\mathrm{g}}\right)^{1 /(s-1)}$ is a dimensionless integration variable, and we defined the probability distribution $\nu(\xi)=2(s-1) \xi^{4 s-7} \exp \left(-\xi^{2(s-1)}\right) / \Gamma[(2 s-3) /(s-1)]$ as well as the auxiliary function

$$
D(x)=\frac{e^{-x^{2}}}{x} \int_{0}^{x} d \xi e^{\xi^{2}} .
$$

The function $D(x)$ is symmetric and monotonically decreasing, with its maximum value $D(x=0)=1$, so that the decoherence function fulfills $\eta(0)=1$. For large arguments it decreases as $D(x) \sim 1 / x^{2}$. We remark that it is related to Dawson's integral $F(x)$ by $D(x)=F(x) / x$ [48.

The function $\nu(\xi)$ is sharply peaked near its mean $\xi_{s}=$ $\Gamma[2-1 / 2(s-1)] / \Gamma[2-1 /(s-1)]$, so that the function $D(x)$ is approximately linear in this region. This allows us to approximate the integral (21) to obtain the decoherence function

$$
\eta(\mathbf{R}) \simeq D\left[\frac{\xi_{s} p_{\mathrm{g}} \theta_{*}\left(p_{\mathrm{g}}\right)|\mathbf{R}|}{2 \hbar}\right] .
$$

The decoherence function describes the decay of the coherences by a single scattering event [41]. Its characteristic width is given by

$$
w_{\eta}=\frac{2 \hbar}{\xi_{s} p_{\mathrm{g}} \theta_{*}\left(p_{\mathrm{g}}\right)} \propto \sqrt{\left\langle\sigma_{\mathrm{tot}}\left(p_{\mathrm{g}}\right)\right\rangle} \propto p_{\mathrm{g}}^{-1 /(s-1)},
$$

i.e. it decreases with increasing momentum $p_{\mathrm{g}}$, where decoherence is more pronounced. The decoherence function (7) shows the asymptotic behavior $\eta(\mathbf{R}) \rightarrow 0$ for $|\mathbf{R}| \gg w_{\eta}$, i.e. the coherence of distant spatial superpositions gets fully destroyed.

The Fourier transform $\widetilde{\eta}(\mathbf{P})$ of the decoherence function gives the distribution of momentum kicks [41. In the present case, it can be evaluated explicitly as

$$
\begin{aligned}
\widetilde{\eta}(\mathbf{P}) & =\frac{1}{(2 \pi \hbar)^{3}} \int_{\mathbb{R}^{3}} d^{3} \mathbf{R} \eta(\mathbf{R}) e^{-i \mathbf{R} \cdot \mathbf{P} / \hbar} \\
& =\frac{1}{2 \pi|\mathbf{P}|}\left(\frac{w_{\eta}}{2 \hbar}\right)^{2} \exp \left[-\left(\frac{|\mathbf{P}| w_{\eta}}{2 \hbar}\right)^{2}\right],
\end{aligned}
$$

which involves the most probable transferred momentum $\sqrt{2} \hbar / w_{\eta}$. Note that the singularity of $\widetilde{\eta}(\mathbf{P})$ at $\mathbf{P}=0$ reflects the fact that the decoherence function (23) is not normalizable. Nevertheless, its Fourier transform (25) is normalized in accordance with $\eta(0)=1$.

For example, in the case of the dipole-induced dipole interaction, the width of the decoherence function $w_{\eta} \approx$ $0.6 \sqrt{\left\langle\sigma_{\text {tot }}\left(p_{\mathrm{g}}\right)\right\rangle}$ is typically in the of range nanometers. In the case of helium atoms, $\alpha_{0} / 4 \pi \varepsilon_{0}=0.2 \AA^{3}$, moving with $p_{\mathrm{g}} / m=10^{3} \mathrm{~m} / \mathrm{s}$, and the molecular dipole moment $d_{0}=5$ Debye, one obtains $w_{\eta} \simeq 0.5 \mathrm{~nm}$. 


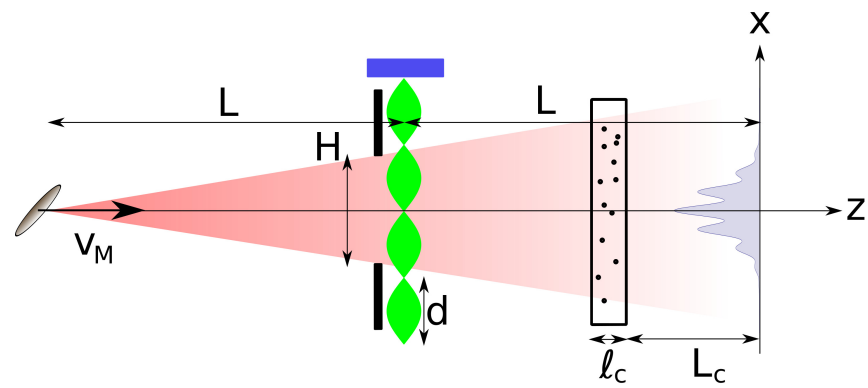

Figure 1. Schematic of a far-field interference experiment with a dedicated collision chamber. The molecules are emitted with longitudinal velocity $v_{M}$ from a point-like source $(z=0)$, propagate the distance $L$ to the grating $(z=L)$, and are detected on the screen $(z=2 L)$ after traversing the collision chamber of width $\ell_{\mathrm{c}}$ placed at the distance $L_{\mathrm{c}}$ in front of the screen.

\section{APPLICATION TO FAR-FIELD MATTER-WAVE INTERFEROMETRY}

In order to illustrate the results of the previous sections, we consider a far-field matter-wave experiment with massive molecules. The particles are emitted with longitudinal velocity $\mathbf{v}_{\mathrm{M}}=v_{\mathrm{M}} \mathbf{e}_{z}$ from a point-like source, propagate freely over the distance $L$ to the diffraction grating with period $d$, and then propagate again for the distance $L$ to the detection screen [2], see Fig. 1]. Since the longitudinal kinetic energy exceeds the average interaction strength as well as the transverse kinetic energy, the interaction with the grating can be described in the eikonal approximation and it suffices to consider the transverse state [49].

We choose our coordinate system such that $x$ denotes the grating axis and $z$ is the flight direction. The $y$ dependence can be neglected in most matter-wave experiments since the extension of the grating in this direction is much larger than the spatial coherence of the particle. In the case of a point source, the interference pattern at the screen can be given as 50 .

$$
w_{0}(x) \propto\left|\int_{-\infty}^{\infty} d x^{\prime} \exp \left[-i \frac{2 \pi x^{\prime}\left(x-x^{\prime}\right)}{d \Delta x}\right] t\left(x^{\prime}\right)\right|^{2}
$$

up to an irrelevant prefactor. Here $\Delta x=2 \pi \hbar L / d M v_{\mathrm{M}}$ is the separation of diffraction peaks in the far field, $H^{2} / d \Delta x \ll 1$, and we defined the grating transformation $t(x)$, which describes the passage through the grating in the eikonal approximation, $\rho \rightarrow \hat{t} \rho \hat{t}^{\dagger}$. In what follows, we consider a far-detuned laser grating of width $H, t(x)=\exp \left[i \phi_{0} \cos ^{2}(\pi x / d)\right] \Theta(H / 2-|x|)$, which acts as a pure phase grating with the maximal phase shift $\phi_{0}$. In the far field, $H^{2} \ll d \Delta x$, the interference pattern is given by the modulus squared of the Fourier transform of the grating function $t(x)$. For the sake of a clear presentation, we neglect the influence of photon absorption [51.

For a typical width of the decoherence function 23 , $w_{\eta} \simeq 0.5 \mathrm{~nm}$, the most probable transferred momentum $\sqrt{2} \hbar / w_{\eta}$ according Eq. 25 is much larger than the grating momentum $2 \pi \hbar / d$. For instance, for $d=200 \mathrm{~nm}$, the ratio is $d / \sqrt{2} \pi w_{\eta} \simeq 90$. This implies that collisions occurring far away from the detection screen reduce the signal because a fraction of the molecules are effectively kicked out of the finite detection range while the shape and contrast of the interference pattern are almost not affected. Hence, the effect of collisional decoherence on the signal is best investigated if the collisions take place close to the screen.

Therefore, we consider a collision chamber of width $\ell_{c}$ placed at the distance $L_{\mathrm{c}}$ in front of the detector, see Fig. 1. We take this chamber to be filled with a monoatomic gas of density $n_{\mathrm{g}}$ and temperature $T$, and the interaction between the polar molecule and the gas particles to be described by the dipole-induced dipole interaction. The range of the homogeneous potential (10) can be estimated to be $R_{\mathrm{w}} \simeq\left(2 m C / \hbar^{2}\right)^{1 /(s-2)}$, where $m$ is the mass of the gas particle. For example, for helium atoms, $m=4 \mathrm{amu}$, at room temperature, $p_{\mathrm{g}} / m \simeq 10^{3} \mathrm{~m} / \mathrm{s}$, and a molecule with dipole moment $d_{0}=5 \mathrm{D}$, the characteristic time scale of the scattering process is approximately $\tau_{\mathrm{c}}=m R_{\mathrm{w}} / p_{\mathrm{g}} \simeq 0.7 \mathrm{ps}$. On the other hand, the rotational period of a linear rigid molecule of mass $M=10^{3}$ amu and length $3 \mathrm{~nm}$ which has the internal temperature $T_{\mathrm{M}}=1000 \mathrm{~K}$, is approximately $\tau_{\text {rot }} \simeq 40 \mathrm{ps}$, and thus $\tau_{\mathrm{c}} / \tau_{\text {rot }} \simeq 50$. Hence, the molecule is almost static during the interaction and the sudden approximation is well justified.

It is shown in Appendix C that the interference pattern can be calculated by

$$
w(x)=e^{-\gamma \ell_{c} / v_{\mathrm{M}}}\left[w_{0}(x)+\int_{-\infty}^{\infty} d x^{\prime} w_{0}\left(x^{\prime}\right) h\left(x-x^{\prime}\right)\right],
$$

where we defined

$$
\begin{aligned}
h(x) & =\int_{-\infty}^{\infty} \frac{d q}{2 \pi \hbar} e^{-i q x / \hbar} \\
& \times\left\{\exp \left[\frac{\gamma}{v_{\mathrm{M}}} \int_{0}^{\ell_{\mathrm{c}}} d z \eta\left(\frac{q\left(L_{\mathrm{c}}+z\right)}{M v_{\mathrm{M}}}\right)\right]-1\right\} .
\end{aligned}
$$

We note that the relation (27) preserves the normalization of $w(x)$. The first term on the right-hand side of Eq. (27) describes the local signal loss, while the second term describes the modification of the shape of the interference pattern due to collisions. If the collision chamber is far away from the detection screen, $L_{\mathrm{c}} \gg L w_{\eta} / d$, the first term dominates the interference signal and the intensity is locally reduced but the shape of the fringes remains unchanged.

In Fig. 2 we show the influence of the collision chamber on the far-field interference pattern of a polar molecule diffracted from a pure phase grating as a function of the distance $L_{\mathrm{c}}$ between the detection screen and the chamber. The collisions with gas atoms induce a positiondependent underground. The width $\Delta I$ of this under- 


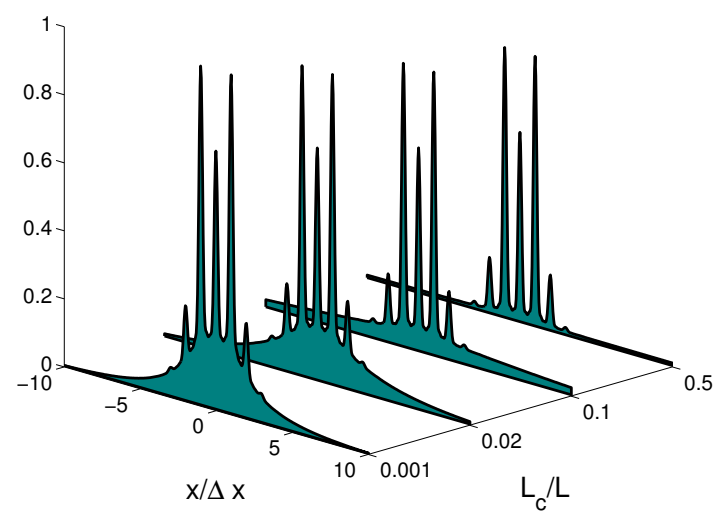

Figure 2. Far-field interference pattern of a polar molecule in the presence of a collision chamber of width $\ell_{\mathrm{c}} / L=0.05$, which is placed in front of the detector at the distances $L_{\mathrm{c}} / L=0.001,0.02,0.1$ and 0.5 . The molecules of mass 840 amu move with velocity $v_{\mathrm{M}}=50 \mathrm{~m} / \mathrm{s}$, and have a dipole moment of 5 Debye. The collision chamber is filled with Helium gas at $300 \mathrm{~K}$ and $5 \mathrm{mPa}$. The atomic polarizability of Helium atoms is taken to be [52] $\alpha / 4 \pi \varepsilon_{0}=0.2 \AA^{3}$. The phase shift and the width of the diffraction grating are chosen to be $\phi_{0}=\pi$ and $H / d=5$.

ground can be estimated assuming that the collisions occur exactly in the middle of the collision chamber,

$$
\frac{\Delta I}{\Delta x} \simeq \frac{d}{\sqrt{2} \pi w_{\eta}} \frac{L_{\mathrm{c}}+\ell_{\mathrm{c}} / 2}{L} .
$$

This relation reflects the fact that in order to investigate the effect of decoherence, the distance between the collision chamber and the detector must be of the order $L_{\mathrm{c}} / L \simeq w_{\eta} / d$.

The fact that the exponential reduction of the signal visibility is proportional to the scattering rate $\gamma$ allows measuring the relative interaction strengths $C^{(1)} / C^{(2)}$ by performing the experiment for two different molecules. For example, if the dominant interaction for both molecules is the dipole-induced dipole interaction, the ratio of the scattering rates obtained for the molecules is $\gamma_{\mathrm{d}-\mathrm{id}}^{(1)} / \gamma_{\mathrm{d}-\mathrm{id}}^{(2)}=\left(d_{0}^{(1)} / d_{0}^{(2)}\right)^{4 / 5}$. This can be used as a measurement of molecular dipole moments in the gas phase.

\section{CONCLUSION}

We derived the master equation for the center-of-mass collisional decoherence of a slowly rotating polar molecule in a thermal gas. Based on the orientationally averaged differential and total scattering cross section of a single collision, we calculated the decoherence function and the scattering rate for the case that the orientation is homogeneously distributed. The decoherence function describes the decay of the coherences and provides the most probable transferred momentum. In the case of the dipole-induced dipole interaction, its width is of the same order of magnitude as the square root of the total scattering cross section.

As an application, we considered a far-field matterwave experiment with an additional collision chamber placed between the grating and the detector and filled with a mono-atomic gas. If the chamber is far away from the detection screen, the interference intensity decreases exponentially with the number of scattering events. Comparing the scattering rate of two different molecules may then allow one to measure the relative strength of molecule-gas interaction. In the case that both molecules are polar, one can thus extract their relative dipole moments. On the other hand, if the collision chamber is placed closer to the detection screen, one starts to observe the modification of the shape of the interference fringes due to collisional decoherence.

\section{ACKNOWLEDGMENTS}

We acknowledge support from the European Commission within NANOQUESTFIT (Contract No. 304886).

\section{Appendix A: Influence of the anisotropy of the thermal gas distribution}

We consider here the angular integration required to evaluate the decoherence function (7), i.e.,

$$
\begin{aligned}
I= & \int_{S_{2}} d^{2} \mathbf{n} \int_{S_{2}} d^{2} \mathbf{n}^{\prime} \mu\left(\left|\mathbf{p}+\mathbf{p}_{0}\right|\right)\left\langle\left|f\left(p, \mathbf{n} \cdot \mathbf{n}^{\prime}\right)\right|^{2}\right\rangle \\
& \times \exp \left[i \zeta \mathbf{u} \cdot\left(\mathbf{n}-\mathbf{n}^{\prime}\right)\right],
\end{aligned}
$$

with the unit vector $\mathbf{u}=\left(\mathbf{R}_{2}-\mathbf{R}_{1}\right) /\left|\mathbf{R}_{2}-\mathbf{R}_{1}\right|$ and the constant $\zeta=\left|\mathbf{R}_{2}-\mathbf{R}_{1}\right| p / \hbar$. If the momentum shift $\left|\mathbf{p}_{0}\right|=m v_{\mathrm{M}}$ is small in comparison to the most probable momentum $p_{\mathrm{g}}$ in the thermal distribution $\mu(|\mathbf{p}|)$, one can expand

$$
\begin{aligned}
\mu\left(\left|\mathbf{p}+\mathbf{p}_{0}\right|\right)= & \mu(p)+\left(\mathbf{p}_{0} \cdot \mathbf{n}\right) \partial_{p} \mu(p) \\
& \times\left[1+\mathcal{O}\left(\frac{m v_{\mathrm{M}}}{p_{\mathrm{g}}}\right)\right],
\end{aligned}
$$

where we used that $\mathbf{p}=p \mathbf{n}$. The zero-th order contribution is spherically symmetric, and the integration result is independent of the direction $\mathbf{u}$, i.e.,

$$
\begin{aligned}
I_{0}= & 8 \pi^{2} \mu(p) \int_{0}^{\pi} d \theta \sin \theta\left\langle|f(p, \cos \theta)|^{2}\right\rangle \\
& \times \operatorname{sinc}\left[2 \zeta \sin \left(\frac{\theta}{2}\right)\right],
\end{aligned}
$$


where $\cos \theta=\mathbf{n} \cdot \mathbf{n}^{\prime}$. The first-order contribution is given by

$$
\begin{aligned}
I_{1}= & {\left[\partial_{p} \mu(p)\right] \mathbf{p}_{0} \cdot \int_{S_{2}} d^{2} \mathbf{n} \int_{S_{2}} d^{2} \mathbf{n}^{\prime} \mathbf{n}\left\langle\left|f\left(p, \mathbf{n} \cdot \mathbf{n}^{\prime}\right)\right|^{2}\right\rangle } \\
& \times \exp \left[i \zeta \mathbf{u} \cdot\left(\mathbf{n}-\mathbf{n}^{\prime}\right)\right] .
\end{aligned}
$$

Simultaneously substituting $\mathbf{n} \rightarrow-\mathbf{n}$ and $\mathbf{n}^{\prime} \rightarrow-\mathbf{n}^{\prime}$ yields that the real part of $I_{1}$ is equal to zero. On the other hand, the imaginary part of $I_{1}$ can be expressed as

$$
\frac{I_{1}-I_{1}^{*}}{2 i} \propto \mathbf{p}_{0} \cdot \nabla_{\mathbf{u}} I_{0}=0
$$

since $I_{0}$ is independent of the direction $\mathbf{u}$. Thus we demonstrated that the linear contribution vanishes and the integral A1 can be written as

$$
I=I_{0}\left[1+\mathcal{O}\left(\frac{m^{2} v_{\mathrm{M}}^{2}}{p_{\mathrm{g}}^{2}}\right)\right]
$$

\section{Appendix B: The small angle differential scattering cross section}

Here, we derive the small angle differential cross section from Eq. (11) by expanding the plane wave up to second order in $\mathbf{n}_{\perp} \cdot \mathbf{b}$ [27]. For this purpose it is beneficial to use spherical coordinates: The outgoing momentum points into the direction $\mathbf{n}^{\prime}=\mathbf{n}^{\prime}(\theta, \phi)$, where $\theta$ and $\phi$ denote the polar and azimuthal angles and, similarly, $\mathbf{m}=\mathbf{m}(\beta, \alpha)$. In addition, $\varphi$ is the angle between $\mathbf{b}$ and the $x$ axis.

In what follows, we will employ the following two approximations:

$$
\frac{1}{2 \pi} \int_{0}^{2 \pi} d \phi\left(1+c \cos ^{2} \phi\right)^{\mu} \approx\left(1+\frac{c}{2}\right)^{\mu}
$$

where $0 \leq c \lesssim 3$ and $0 \leq \mu \leq 1$, and

$$
\frac{1}{2} \int_{0}^{\pi} d \phi \sin \phi\left(1+c \cos ^{2} \phi\right)^{\mu} \approx\left(1+\frac{c}{3}\right)^{\mu},
$$

where $|c|<1$ and $0 \leq \mu \leq 3$. The relative error does not exceed $3 \%$ for the parameter range given above.

For the discussion to follow, it is important to note that the $z$ integration in Eq. (11) can be carried out explicitly to give

$$
\begin{aligned}
& \int_{-\infty}^{\infty} d z V\left(\sqrt{b^{2}+z^{2}}, \frac{\left(\mathbf{b}+z \mathbf{e}_{z}\right) \cdot \mathbf{m}}{\sqrt{b^{2}+z^{2}}}\right) \\
& =-\frac{\sqrt{\pi} C}{b^{s-1}} \frac{\Gamma[(s-1) / 2]}{\Gamma(s / 2)}[G(\beta, \varphi-\alpha)]^{(s-1) / 2},
\end{aligned}
$$

where

$$
G(\beta, \varphi)=\left(1+\frac{a}{s} \cos ^{2} \beta+\frac{a(s-1)}{s} \cos ^{2} \varphi \sin ^{2} \beta\right)^{2 /(s-1)}
$$

Then, the zeroth order contribution $f_{0}\left(p, \mathbf{n}^{\prime} ; \mathbf{m}\right)$ can be evaluated with the help of the integrals [53.

$$
\begin{aligned}
& \int_{0}^{\infty} d b b^{m} \sin ^{2}\left(\frac{A}{2 b^{s-1}}\right)=\frac{A^{(m+1) /(s-1)}}{2(m+1)} \\
& \times \Gamma\left(\frac{s-m-2}{s-1}\right) \sin \left(\frac{\pi}{2} \frac{s-m-2}{s-1}\right) \\
& \int_{0}^{\infty} d b b^{m} \sin \left(\frac{A}{b^{s-1}}\right)=\frac{A^{(m+1) /(s-1)}}{(m+1)} \\
& \times \Gamma\left(\frac{s-m-2}{s-1}\right) \cos \left(\frac{\pi}{2} \frac{s-m-2}{s-1}\right),
\end{aligned}
$$

for $A, m \in \mathbb{R}$, and $1<(m+s) /(s-1)<3$ as well as $0<(m+s) /(s-1)<2$, respectively. Thus, one obtains

$$
\begin{aligned}
f_{0}\left(p, \mathbf{n}^{\prime} ; \mathbf{m}\right) & =\frac{p}{4 \pi \hbar} \exp \left(\frac{i \pi}{2} \frac{s-3}{s-1}\right) \Gamma\left(\frac{s-3}{s-1}\right) \\
& \times\left(\frac{m \sqrt{\pi} C}{\hbar p} \frac{\Gamma[(s-1) / 2]}{\Gamma(s / 2)}\right)^{2 /(s-1)} \\
& \times \int_{0}^{2 \pi} d \varphi G(\beta, \varphi) .
\end{aligned}
$$

In a similar fashion, the linear contribution vanishes due to the symmetry of the integrand. The leading order correction is thus the quadratic term, which can be evaluated analogously to the above calculation,

$$
\begin{aligned}
& f_{2}\left(p, \mathbf{n}^{\prime} ; \mathbf{m}\right) \\
& =-\frac{p}{16 \pi \hbar} \exp \left(\frac{i \pi}{2} \frac{s-5}{s-1}\right) \Gamma\left(\frac{s-5}{s-1}\right) \\
& \times\left(\frac{p \theta}{\hbar}\right)^{2}\left(\frac{m \sqrt{\pi} C}{\hbar p} \frac{\Gamma[(s-1) / 2]}{\Gamma(s / 2)}\right)^{4 /(s-1)} \\
& \times\left[\sin ^{2}(\phi-\alpha) \int_{0}^{2 \pi} d \varphi G^{2}(\beta, \varphi)+\cos (2 \phi-2 \alpha)\right. \\
& \left.\times \int_{0}^{2 \pi} d \varphi \cos ^{2} \varphi G^{2}(\beta, \varphi)\right] .
\end{aligned}
$$

Adding the two contributions and orientationally averaging the squared absolute value finally gives Eq. (15, where the constants $A$ and $\theta_{*}$ are given by

$$
\begin{aligned}
A & =\left(\frac{p \sigma_{0}(p)}{4 \pi \hbar}\right)^{2}\left[1+\tan ^{2}\left(\frac{\pi}{s-1}\right)\right] h_{1}(a), \\
\theta_{*} & =\frac{\hbar}{p} \sqrt{\frac{8 \pi}{\sigma_{0}(p)} \Gamma\left(\frac{s-3}{s-1}\right)\left[\Gamma\left(\frac{s-5}{s-1}\right) h_{2}(a)\right]^{-1 / 2}} .
\end{aligned}
$$

Here, we defined the two functions

$$
h_{1}(a)=\frac{1}{8 \pi^{2}} \int_{0}^{\pi} d \beta \sin \beta\left[\int_{0}^{2 \pi} d \varphi G(\beta, \varphi)\right]^{2},
$$

and

$$
\begin{aligned}
h_{2}(a)= & \frac{1}{4 \pi^{2} h_{1}(a)} \int_{0}^{\pi} d \beta \int_{0}^{2 \pi} d \varphi d \varphi^{\prime} \sin \beta \\
& \times G(\beta, \varphi) G^{2}\left(\beta, \varphi^{\prime}\right) .
\end{aligned}
$$


With the approximations presented above, the two functions (B11) and $(\mathrm{B} 12)$ can be written as

$$
\begin{aligned}
& h_{1}(a)=\left(1+\frac{a}{3}\right)^{4 /(s-1)}, \\
& h_{2}(a)=\left(1+\frac{a}{3}\right)^{2 /(s-1)},
\end{aligned}
$$

which finally gives Eqs. 16 and 17, respectively.

\section{Appendix C: Molecular density distribution in far-field interferometry}

Here, we present the theoretical description of the molecular c.m. dynamics in a far-field matter-wave experiment with a collision chamber. A typical far-field setup consists of a molecular source $(z=0)$, a diffraction grating $\left(z=L_{1}\right)$ and a screen $\left(z=L_{1}+L_{2}\right)$. Since the motional c.m. state of the molecule is separable in $x, y$ and $z$ directions at all times [41, one can reduce the problem to a single dimension and it suffices to treat the dynamics in the $x$-direction. The motion in the $z$ direction is then approximated by replacing $z=v_{\mathrm{M}} t$.

The interference pattern can be conveniently expressed with the help of the Wigner function $w(x, p)$ or, equivalently, with its characteristic function [54]

$$
\chi(s, q)=\int_{-\infty}^{\infty} \mathrm{d} x \int_{-\infty}^{\infty} \mathrm{d} p w(x, p) e^{i(q x-p s) / \hbar} .
$$

In particular, the solution of the master equation for collisional decoherence,

$$
\begin{aligned}
\partial_{t} \rho\left(x, x^{\prime}\right)= & \frac{1}{i \hbar}\left\langle x|[\mathrm{H}, \rho]| x^{\prime}\right\rangle \\
& +\gamma(t)\left[\eta\left(x-x^{\prime}\right)-1\right] \rho\left(x, x^{\prime}\right),
\end{aligned}
$$

with the free Hamiltonian $\mathrm{H}$ and the time-dependent scattering rate $\gamma(t)$ can be given in terms of the characteristic function as

$$
\begin{aligned}
& \chi_{t}(s, q)=\chi_{0}\left(s-\frac{q t}{M}, q\right) \\
& \times \exp \left\{\int_{0}^{t} d \tau \gamma(\tau)\left[\eta\left(s-\frac{q}{M}(t-\tau)\right)-1\right]\right\} .
\end{aligned}
$$

The state after time $t, \chi_{t}$, is described by multiplying the initial state $\chi_{0}$ by an exponential reduction factor due to decoherence followed by the shearing transformation $s \rightarrow s-q t / M$. The time dependence of the collision rate $\gamma$ may account for a spatially varying gas density, $n_{\mathrm{g}}\left(z=v_{\mathrm{M}} t\right)$.

If the scattering occurs in a chamber placed between the diffraction grating and the detector, the state in front of the detector reads

$$
\begin{aligned}
\chi_{t_{1}+t_{2}}(s, q) & =\chi_{t_{1}}^{\prime}\left(s-\frac{q}{M} t_{2}, q\right) \\
& \times \exp \left\{\int_{0}^{t_{2}} d \tau \gamma\left(\tau+t_{1}\right)\right. \\
& \left.\times\left[\eta\left(s-\frac{q}{M}\left(t_{2}-\tau\right)\right)-1\right]\right\} .
\end{aligned}
$$

Here, $t_{1}$ and $t_{2}$ denote the time of flight between source and grating and between grating and detector, respectively, and $\chi_{t_{1}}^{\prime}(s, q)$ is the state immediately after the diffraction grating. The density distribution on the screen is then given by

$$
\begin{aligned}
w(x)= & \frac{1}{2 \pi \hbar} \int_{-\infty}^{\infty} d q e^{-i q x / \hbar} \chi_{t_{1}+t_{2}}(0, q) \\
= & \exp \left[\int_{0}^{t_{2}} d \tau \gamma\left(\tau+t_{1}\right)\right] \\
& \times\left[w_{0}(x)+\int_{-\infty}^{\infty} d x^{\prime} w_{0}\left(x^{\prime}\right) h\left(x-x^{\prime}\right)\right]
\end{aligned}
$$

where $w_{0}(x)$ is the interference pattern in absence of decoherence and

$$
\begin{gathered}
h(x)=\frac{1}{2 \pi \hbar} \int_{-\infty}^{\infty} d q e^{-i q x / \hbar} \\
\times\left[\exp \left\{\int_{0}^{t_{2}} d \tau \gamma\left(\tau+t_{1}\right) \eta\left[\frac{q}{M}\left(t_{2}-\tau\right)\right]\right\}-1\right] .
\end{gathered}
$$

Assuming that the rate $\gamma(t)$ is constant in the chamber and zero outside yields Eq. 27.
[1] W. Schöllkopf and J. P. Toennies, Nondestructive mass selection of small van der Waals clusters, Science 266, 1345-1348 (1994).

[2] M. Arndt, O. Nairz, J. Vos-Andreae, C. Keller, G. van der Zouw, and A. Zeilinger, Wave-particle duality of C(60) molecules, Nature (London) 401, 680-2 (1999).

[3] B. Brezger, L. Hackermüller, S. Uttenthaler, J. Petschinka, M. Arndt, and A. Zeilinger, Matterwave interferometer for large molecules, Phys. Rev. Lett. 88, 100404 (2002).

[4] A. A. Milner, A. Korobenko, J. W. Hepburn, and V. Mil- ner, Effects of ultrafast molecular rotation on collisional decoherence, Phys. Rev. Lett. 113, 043005 (2014).

[5] S. Eibenberger, S. Gerlich, M. Arndt, J. Tüxen, and M. Mayor, Electric moments in molecule interferometry, N. J. Phys. 13, 043033 (2011).

[6] S. Gerlich, S. Eibenberger, M. Tomandl, S. Nimmrichter, K. Hornberger, P. J. Fagan, J. Tüxen, M. Mayor, and M. Arndt, Quantum interference of large organic molecules, Nat. Commun. 2, 263 (2011).

[7] N. Dörre, J. Rodewald, P. Geyer, B. von Issendorff, P. Haslinger, and M. Arndt, Photofragmentation beam 
splitters for matter-wave interferometry, Phys. Rev. Lett. 113, 233001 (2014).

[8] P. Geyer, U. Sezer, J. Rodewald, L. Mairhofer, N. Dörre, P. Haslinger, S. Eibenberger, C. Brand, and M. Arndt, Perspectives for quantum interference with biomolecules and biomolecular clusters, Phys. Scr. 91, 063007 (2016).

[9] M. A. Schlosshauer, Decoherence and the Quantum-toClassical Transition (Springer, Berlin, 2008).

[10] E. Joos, H. Zeh, C. Kiefer, D. Giulini, J. Kupsch, and I. Stamatescu, Decoherence and the Appearance of a Classical World in Quantum Theory (Springer, Berlin, 2002).

[11] W. H. Zurek, Decoherence, einselection, and the quantum origins of the classical, Rev. Mod. Phys. 75, 715-775 (2003).

[12] J. Trost and K. Hornberger, Hund's paradox and the collisional stabilization of chiral molecules, Phys. Rev. Lett. 103, 023202 (2009).

[13] I. Gonzalo and P. Bargueño, Stabilization of chiral molecules by decoherence and environment interactions in the gas phase, Phys. Chem. Chem. Phys. 13, 1713017134 (2011).

[14] P. J. Coles, V. Gheorghiu, and R. B. Griffiths, Consistent histories for tunneling molecules subject to collisional decoherence, Phys. Rev. A 86, 042111 (2012).

[15] S. L. Adler, Quantum theory as an emergent phenomenon: The statistical mechanics of matrix models as the precursor of quantum field theory (Cambridge University Press, Cambridge, UK, 2004).

[16] A. Bassi, K. Lochan, S. Satin, T. P. Singh, and H. Ulbricht, Models of wave-function collapse, underlying theories, and experimental tests, Rev. Mod. Phys. 85, 471 (2013).

[17] M. Arndt and K. Hornberger, Testing the limits of quantum mechanical superpositions, Nat. Phys. 10, 271-277 (2014).

[18] K. Hornberger, S. Uttenthaler, B. Brezger, L. Hackermüller, M. Arndt, and A. Zeilinger, Collisional decoherence observed in matter wave interferometry, Phys. Rev. Lett. 90, 160401 (2003).

[19] I. F. Tenney, M. Artamonov, T. Seideman, and P. H. Bucksbaum, Collisional decoherence and rotational quasirevivals in asymmetric-top molecules, Phys. Rev. A 93, 013421 (2016).

[20] K. Hornberger, Master equation for a quantum particle in a gas, Phys. Rev. Lett. 97, 060601 (2006).

[21] B. Vacchini and K. Hornberger, Quantum linear Boltzmann equation, Phys. Rep. 478, 71-120 (2009).

[22] J. R. Taylor, Scattering Theory: The Quantum Theory of Nonrelativistic Collisions, (Dover, Mineola, New York, 2000).

[23] A. Smirne and B. Vacchini, Quantum master equation for collisional dynamics of massive particles with internal degrees of freedom, Phys. Rev. A 82, 042111 (2010).

[24] C. Hemming and R. Krems, Collisional decoherence of internal-state superpositions in a trapped ultracold gas, Phys. Rev. A 81, 052701 (2010).

[25] H. G. Bennewitz, K. Kramer, W. Paul, and J. Toennies, Messung der Anisotropie des van der Waals-Potentials durch Streuung von Molekülen in definiertem Quantenzustand, Z. Phys. 177, 84-110 (1964).

[26] K. H. Kramer and R. B. Bernstein, Sudden approximation applied to rotational excitation of molecules by atoms, I: Low-angle scattering, J. Chem. Phys. 40, 200-
203 (1964).

[27] M. S. Child, Molecular Collision Theory (Academic Press, London, 1974).

[28] R. Helbing and H. Pauly, Differentielle Streuquerschnitte bei der Wechselwirkung neutraler Moleküle Messung der Kleinwinkelstreuung, Z. Phys. 179, 16 (1964).

[29] D. Patterson, E. Tsikata, and J. M. Doyle, Cooling and collisions of large gas phase molecules, Phys. Chem. Chem. Phys. 12, 9736-9741 (2010).

[30] N. R. Hutzler, H.-I. Lu, and J. M. Doyle, The buffer gas beam: An intense, cold, and slow source for atoms and molecules, Chem. Rev. 112, 4803-4827 (2012).

[31] S. N. Vogels, J. Onvlee, A. von Zastrow, G. C. Groenenboom, A. van der Avoird, and S. Y. van de Meerakker, High-resolution imaging of velocity-controlled molecular collisions using counterpropagating beams, Phys. Rev. Lett. 113, 263202 (2014).

[32] K. Miyajima, M. B. Knickelbein, and A. Nakajima, Stern-Gerlach study of multidecker lanthanidecyclooctatetraene sandwich clusters, J. Phys. Chem. A 112, 366-375 (2008).

[33] S. Heiles, S. Schäfer, and R. Schäfer, On the rotational temperature and structure dependence of electric field deflection experiments: A case study of germanium clusters, J. Chem. Phys. 135, 034303 (2011).

[34] H. Pauly, Atom, Molecule, and Cluster Beams II: Cluster Beams, Fast and Slow Beams, Accessory Equipment and Applications, Vol. 32 (Springer, Berlin, 2013).

[35] J. J. Sakurai, Modern Quantum Mechanics (Addison Wesley, Reading, MA, 1993).

[36] D. M. Brink and G. R. Satchler, Angular Momentum, 3rd ed. (Clarendon Press, Oxford, UK, 1994).

[37] K. Hornberger, Monitoring approach to open quantum dynamics using scattering theory, Europhys. Lett. 77, 50007 (2007).

[38] K. Hornberger and B. Vacchini, Monitoring derivation of the quantum linear Boltzmann equation, Phys. Rev. A 77, 022112 (2008).

[39] A. M. Arthurs and A. Dalgarno, The theory of scattering by a rigid rotator, Proc. Roy. Soc. (London) A 256, 540551 (1960).

[40] B. A. Stickler and K. Hornberger, Molecular rotations in matter-wave interferometry, Phys. Rev. A 92, 023619 (2015).

[41] K. Hornberger, J. E. Sipe, and M. Arndt, Theory of decoherence in a matter wave Talbot-Lau interferometer, Phys. Rev. A 70, 053608 (2004).

[42] A. J. Stone, The Theory of Intermolecular Forces (Oxford University Press, Oxford, UK, 1997).

[43] L. I. Schiff, Approximation method for high-energy potential scattering, Phys. Rev. 103, 443-453 (1956).

[44] L. D. Landau and E. M. Lifshitz, Course of Theoretical Physics, Volume 3: Quantum Mechanics, 3rd ed. (Pergamon, Oxford, UK, 1981).

[45] K. E. Mount, Potential scattering cross sections in the semiclassical limit, J. Phys. B. 6, 1397 (1973).

[46] M. V. Berry and K. E. Mount, Semiclassical approximations in wave mechanics, Rep. Prog. Phys. 35, 315 (1972).

[47] G. W. F. Drake, editor, Springer Handbook of Atomic, Molecular, and Optical Physics, 2nd ed. (Springer, New York, 2005).

[48] M. Abramowitz and I. A. Stegun, Handbook of Mathematical Functions: with Formulas, Graphs, and Mathe- 
matical Tables (Dover, New York, 1965).

[49] S. Nimmrichter and K. Hornberger, Theory of near-field matter-wave interference beyond the eikonal approximation, Phys. Rev. A 78, 023612 (2008).

[50] M. Born and E. Wolf, Principles of Optics: Electromagnetic Theory of Propagation, Interference and Diffraction of Light, 7th ed. (Cambridge university press, Cambridge, UK, 1999).

[51] J. P. Cotter, S. Eibenberger, L. Mairhofer, X. Cheng, P. Asenbaum, M. Arndt, K. Walter, S. Nimmrichter, and $\mathrm{K}$. Hornberger, Coherence in the presence of absorption and heating in a molecule interferometer, Nat. Commun.
6, $7336(2015)$.

[52] W. Haynes, editor, Handbook of Chemistry and Physics (CRC Press, Boca Raton, FL, 2012).

[53] I. S. Gradshteyn and I. M. Ryzhik, Table of Integrals, Series, and Products, 7th ed. (Academic Press, Orlando, FL, 2014).

[54] J. Bateman, S. Nimmrichter, K. Hornberger, and H. Ulbricht, Near-field interferometry of a free-falling nanoparticle from a point-like source, Nat. Commun. 5, 4788 (2014). 